\title{
Validated Simultaneous Gradient Ultra-Performance Liquid Chromatographic Quantification of Some Proton Pump Inhibitor Drug Residues in Saudi Pharmaceutical Industrial Wastewater
}

\author{
Sherif A. Abdel-Gawad ${ }^{1,2, *}$, Hany H. Arab ${ }^{3}$ (D) and Alhumaidi B. Alabbas ${ }^{1}$ \\ 1 Pharmaceutical Chemistry Department, College of Pharmacy, Prince Sattam Bin Abdulaziz University, \\ Al Kharj 11942, Saudi Arabia; ab.alabbas@psau.edu.sa \\ 2 Analytical Chemistry Department, Faculty of Pharmacy, Cairo University, Cairo ET-11562, Egypt \\ 3 Department of Pharmacology and Toxicology, College of Pharmacy, Taif University, P.O. Box 11099, \\ Taif 21944, Saudi Arabia; h.arab@tu.edu.sa \\ * Correspondence: sagawad@yahoo.com; Tel.: +966-540-586-921
}

\section{check for} updates

Citation: Abdel-Gawad, S.A.; Arab, H.H.; Alabbas, A.B. Validated

Simultaneous Gradient

Ultra-Performance Liquid

Chromatographic Quantification of

Some Proton Pump Inhibitor Drug Residues in Saudi Pharmaceutical Industrial Wastewater. Molecules 2021, 26, 4358. https://doi.org/10.3390/ molecules26144358

Academic Editor: Yannis Dotsikas

Received: 4 June 2021

Accepted: 16 July 2021

Published: 19 July 2021

Publisher's Note: MDPI stays neutral with regard to jurisdictional claims in published maps and institutional affiliations.

Copyright: (c) 2021 by the authors. Licensee MDPI, Basel, Switzerland. This article is an open access article distributed under the terms and conditions of the Creative Commons Attribution (CC BY) license (https:// creativecommons.org/licenses/by/ $4.0 /)$.

\begin{abstract}
Monitoring and quantification of active pharmaceutical ingredients (APIs) in the environment constitute important and challenging tasks, as they are directly associated with human health. Three commonly used proton pump inhibitors (PPIs), namely, omeprazole sodium (OMP), pantoprazole sodium (PNT), and lansoprazole sodium (LNZ) are well separated and quantified using ultra-performance liquid chromatography (UPLC) in pharmaceutical industrial wastewater. The separation of the studied drugs was performed on a stationary phase with a Waters ${ }^{\mathrm{TM}}$ column $(100 \times 2.1 \mathrm{~mm}, 1.7 \mu \mathrm{m})$. The mobile phase was composed of methanol:0.05 M potassium dihydrogen phosphate buffer (adjusted to $\mathrm{pH} 7.5$ using $\mathrm{NaOH})(50: 50, v / v)$. The elution process was done in gradient mode by changing the relative proportions of the mobile phase components with time to get an optimum separation pattern. The flow rate of the developing system was adjusted to $0.8 \mathrm{~mL} /$ minute. Detection of the separated drugs was performed at $230 \mathrm{~nm}$. The studied drugs were quantified in the concentration range of $10-200 \mathrm{ng} / \mathrm{mL}$ for all drugs. The cited method was fully validated according to the international conference on harmonization (ICH-Q2B) guidelines, then it was applied successfully for quantification of the studied PPIs in real wastewater samples after their solid phase extraction (SPE).
\end{abstract}

Keywords: drug residues; environmental; proton pump inhibitors; UPLC; wastewater

\section{Introduction}

Stomach acid is a natural and valuable chemical contributor to the digestion process. However, in excess or in the wrong place it is a menace, inflaming and irritating the esophagus. Additionally, it may cause heartburn and sometimes contribute to the development of ulcers in the stomach, the duodenum, and the first part of the small intestine. Proton pump inhibitors (PPIs) are a group of medications whose main action is a pronounced and long-lasting reduction in stomach acid production. They are widely prescribed for the treatment of gastroesophageal reflux disease (GERD), acid reflux, heartburn, and stomach ulcers. Additionally, they are considered an important component in the triple therapy of Helicobacter pylori infection [1].

In the last few years, the widespread use of PPIs may have caused their existence in the environment, especially in surface water. Despite many studies having carried out progressive determinations of pharmaceutical compounds in environmental wastewaters and their negative influences on human health, there is no limit value for the presence of these pollutants in soil or natural water [2]. PPIs may cause a lot of adverse effects when reaching the human body. These adverse effects may be mild like headaches, diarrhea, 
constipation, abdominal pain, flatulence, and fever. Long term exposure to PPIs may lead to serious effects, including an increased risk of Clostridium difficile infection in the colon, gastric carcinoid tumor, and osteoporosis-related fractures of the hip, wrist, or spine. Additionally, the increased risk of heart attacks may occur due to long-term exposure to PPIs. The residues of PPIs may reach children from the surrounding environment and may cause serious effects upon long-term exposure. These effects are the increased risk of gastrointestinal and respiratory tract infections, vitamin B12 deficiency, hypomagnesemia, and bone fracture. PPIs are excreted from the body through the urine, which could reach the hospital sewage. Additionally, the studied drugs could be present in the wastewater of pharmaceutical plants and so they could reach surface water easily. Due to the negative influences of these compounds on human health upon long-term exposure, they should not be present in surface water or soil even at very low concentrations. They are regarded as pollutants [3].

Omeprazole sodium (OMP), pantoprazole sodium (PNT), and lansoprazole sodium (LNZ) are three commonly used PPIs in the Saudi market. They are included in this study to monitor their amounts in industrial wastewater.

OMP $\left(\mathrm{C}_{17} \mathrm{H}_{18} \mathrm{~N}_{3} \mathrm{NaO}_{3} \mathrm{~S}\right)$, sodium;5-methoxy-2-[(4-methoxy-3,5-dimethyl pyridine2-yl)methylsulfinyl]benzimidazol-1-ide, decreases the capability of the parietal cells to secrete gastric acid through its action on the $\left(\mathrm{H}^{+} / \mathrm{K}^{+}\right)$-ATPase enzyme [4]. Many techniques have been applied for the quantification of OMP in different samples. It was quantified spectrophotometrically depending on its combination with ion-pairing agents, like bromophenol blue and orange $G$, then the formed reaction products were measured at $408 \mathrm{~nm}$ and $508 \mathrm{~nm}$, respectively [5]. OMP was determined simultaneously with diclofenac potassium [6] or aspirin [7] by using either the simultaneous equation method or zero-order and area under curve methods. High-performance liquid chromatography (HPLC) was applied for the determination of OMP in capsules depending on its separation from the C18 column and UV-detection [4]. Additionally, it was quantified in plasma after its extraction with tertiary butyl methyl ether, separation on a C18 column, and detection using a photodiode array detector [8]. Liquid chromatography coupled with tandem mass spectroscopy (LC-MS/MS) was applied for the determination of OMP in human plasma. It was detected using multiple reaction monitoring (MRM) mode [9]. Ultra-performance liquid chromatography (UPLC) was applied for the determination of OMP, either simultaneously with domperidone in capsules [10] or with its related substances using TOF/MS detection [11]. Meanwhile, UPLC was used for OMP assay in its magnesium blend [12].

PNT $\left(\mathrm{C}_{16} \mathrm{H}_{14} \mathrm{~F}_{2} \mathrm{~N}_{3} \mathrm{NaO}_{4} \mathrm{~S}\right.$ ), sodium;5-(difluoromethoxy)-2-[(3,4-dimethoxypyridin-2yl)methylsulfinyl]benzimidazol-1-ide, was quantified titrimetrically and spectrophotometrically in pharmaceuticals using cerium (IV) sulphate as an oxidimetric agent [13]. It was also determined spectrophotometrically with OMP through complex formation with iron (III) in an aqueous ethanol medium [14]. LC-MS/MS was applied for the determination of PNT using LNZ as an internal standard (IS), either in human plasma [15] or in human urine [16]. The same technique was also applied for the sensitive monitoring of the studied drug in a bioequivalence study [17].

LNZ $\left(\mathrm{C}_{16} \mathrm{H}_{13} \mathrm{~F}_{3} \mathrm{~N}_{3} \mathrm{NaO}_{2} \mathrm{~S}\right)$, sodium;2-[[3-methyl-4-(2,2,2-trifluoromethoxy)pyridin-2yl]methylsulfinyl]benzimidazol-1-ide, is a potent PPI. It was determined spectrophotometrically in capsules and spiked human urine through ion-pair complex formation with bromocresol purple and bromothymol blue [18]. Kinetic spectrophotometry was applied for the quantification of LNZ in a pharmaceutical formulation via its reaction with alkaline potassium permanganate at room temperature [19]. Extractive spectroscopy was applied for the determination of LNZ in pharmaceutical dosage form after its reaction with metanil yellow and methyl orange followed by extraction with chloroform [20]. HPLC was applied for the determination of LNZ with UV-detection [21]. Additionally, the same technique was applied for the quantification of the studied drug with its related impurities [22]. UPLC was applied for the determination of LNZ in commercial pharmaceutical products [23]. Additionally, the same technique was demonstrated for the simultaneous quantification 
of the studied drug with naproxen [24]. LC-MS/MS was applied for the determination of LNZ in human plasma, using esomeprazole as an IS [25].

In the last few years, there has been an enormous increase in Helicobacter pylori infections all over the world. This, in turn, has increased the prescribing rates of PPIs, especially in the Saudi market. OMP, PNT, and LNZ are the most widely and frequently prescribed PPIs in the Saudi market. Many research papers have been published dealing with different pharmaceuticals, including PPIs in the aquatic environment for many regions in the world, especially in Europe [26-29]. OMP, PNT, and LNZ are manufactured by many factories in the Kingdom of Saudi Arabia (KSA). Therefore, there is a big chance for these drugs to reach surface and irrigation water. Accordingly, there is an imminent threat to human health, especially in the regions surrounding the manufacturing factories. Therefore, there is an urgent need to optimize, validate, and apply an analytical method to monitor and quantify these three PPIs in industrial wastewater for that pharmaceutical factory.

UPLC is a chromatographic system that can use a combination of reversed-phase packing substances with a size of $1.7 \mu \mathrm{m}$ and an operation pressure in the $6000-15,000 \mathrm{psi}$ range. It has many advantages over conventional HPLC, including a greater sensitivity as characterized by a greater signal to noise $(\mathrm{S} / \mathrm{N})$ ratio due to the reduction in zone broadening. Additionally, it is characterized by better chromatographic peak resolution and increased speed of analysis. UPLC is characterized by higher sensitivity and selectivity compared to the molecular spectrophotometric methods. Regarding the suggested technique (UPLC), when compared to LC-MS/MS it has comparable sensitivity but with more economic operating expenses. Additionally, UPLC does not require special instrument assembly [30]. After an extensive literature review, no published papers have directly conducted a UPLC determination of the studied PPI drug residues in Saudi pharmaceutical industrial wastewater.

The main goal of this work is to optimize and validate a simple, sensitive, accurate, and reproducible UPLC method to quantify the studied drugs in Saudi pharmaceutical industrial wastewater after their pretreatment using solid-phase extraction (SPE).

\section{Results}

This work demonstrates the capability of selective simultaneous quantification of three commonly used PPI drug residues in pharmaceutical wastewater samples using UPLC in wastewater samples, which in turn, make the environmental monitoring task of the studied drugs, an easy process.

\subsection{Method Optimization}

The best separation pattern was achieved using the stationary phase of the Waters ${ }^{\mathrm{TM}}$ column $(100 \times 2.1 \mathrm{~mm}, 1.7 \mu \mathrm{m})$ and a mobile phase composed of methanol:0.05 M potassium dihydrogen phosphate buffer (adjusted to $\mathrm{pH} 7.5$ using $\mathrm{NaOH})(50: 50, v / v)$. The composition of the mobile phase was changed by increasing the percentage of methanol by $5 \%$ and decreasing the percentage of the buffer by $5 \%$ every $4 \mathrm{~min}$. The mobile phase flow rate was adjusted to $0.8 \mathrm{~mL} / \mathrm{min}$. On the other hand, detection of eluted drugs was performed at $230 \mathrm{~nm}$. The retention times were $14.40 \mathrm{~min}$ for OMP, $14.90 \mathrm{~min}$ for PNT and 15.40 min for LNZ, Figure 1. 


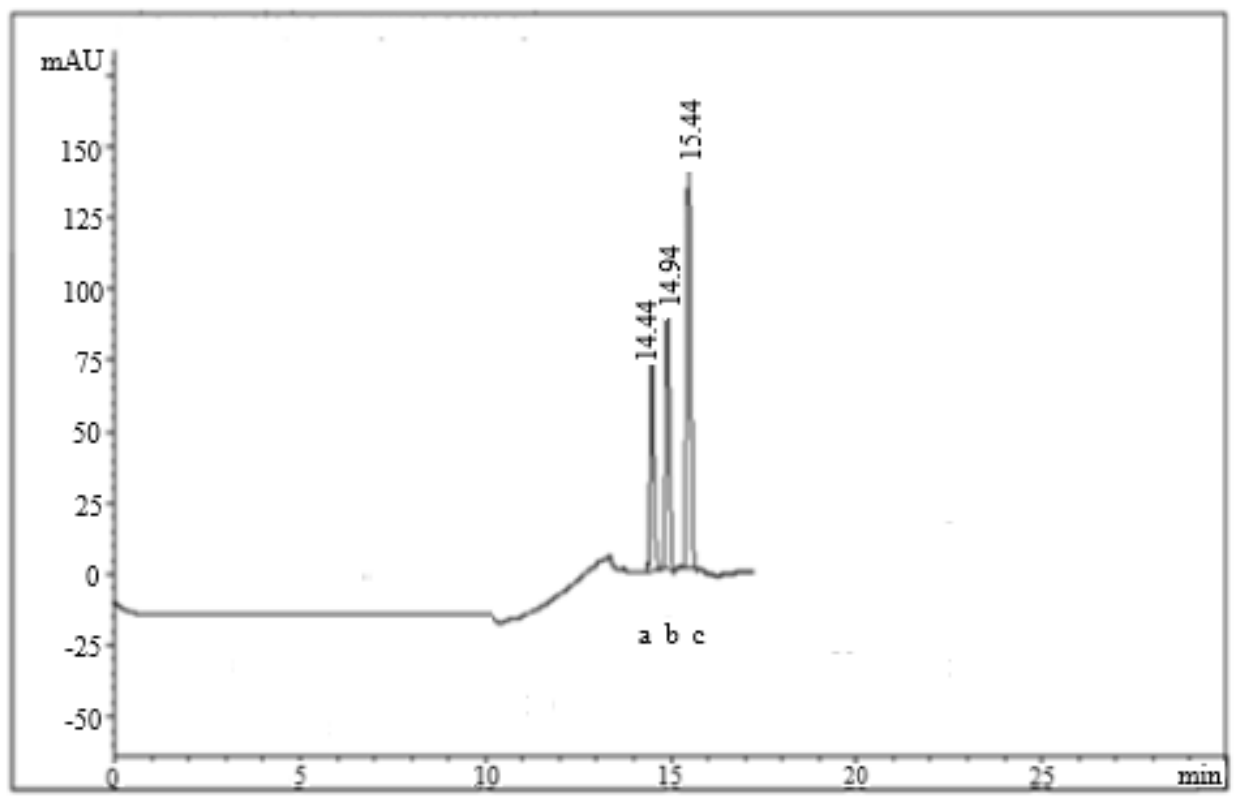

Figure 1. UPL chromatogram for the separation pattern of OMP (a), PNT (b), and LNZ (c) reference materials.

Acceptable system suitability parameters were obtained in Table 1 . The values for the number of theoretical plates $(\mathrm{N})$ and height equivalent to theoretical plates (HETP) confirmed excellent column efficiency. At the same time, good selectivity and resolution were ensured by the resolution factor (Rs). Additionally, the tailing factor value confirmed acceptable peak symmetry.

Table 1. Established system suitability parameters of the UPLC-method.

\begin{tabular}{cccc}
\hline Parameter & OMP & PPZ & LNZ \\
\hline $\mathrm{t}_{\mathrm{R} \text { (min.) }}{ }^{\dagger}(n=3)$ & $14.40 \pm 0.15$ & $14.90 \pm 0.21$ & $15.40 \pm 0.17$ \\
Capacity factor $(\mathrm{K})$ & 71 & 74 & 76 \\
Resolution factor $(\mathrm{Rs})$ & - & 5 & 5 \\
Number of theoretical plates & 331,776 & 355,216 & 379,456 \\
$(\mathrm{~N})$ & $3.1 \times 10^{-5}$ & $2.8 \times 10^{-5}$ & $2.6 \times 10^{-5}$ \\
HETP & 1.04 & 1.04 & 1.04 \\
\hline Tailing factor $(\mathrm{T})$ &
\end{tabular}

${ }^{\dagger}$ Triplicate runs per sample. ${ }^{*}$ Height equivalent to theoretical plates.

\subsection{Method Validation}

The validation was carried out according to ICH-Q2B guidelines [31]. A linear relation was obtained between the peak area and the concentration of the studied drugs in the concentration range $10-200 \mathrm{ng} / \mathrm{mL}$. The following regression equations were computed to be:

$$
\begin{aligned}
& \text { PA }(\mathrm{OMP})=97.116 \mathrm{C}+1.4526 \mathrm{r}=0.9999 \\
& \text { PA }(\mathrm{PPZ})=52.406 \mathrm{C}+9.1226 \mathrm{r}=0.9998 \\
& \text { PA }(\mathrm{LNZ})=94.542 \mathrm{C}+7.1121 \mathrm{r}=0.9997
\end{aligned}
$$

where, PA: peak area. C: Concentration (ng/ mL). r: Correlation coefficient.

The validation sheet showed acceptable accuracy, repeatability, and intermediate precision as demonstrated in Table 2. Assessment of the method's robustness was also done by carrying out a slight change in the composition of the mobile phase, $\mathrm{pH}$, and flow rate. All the results confirmed that there is no significant effect on the proposed method when carrying out these slight changes, which confirmed the robustness of the suggested 
method. The calculated values of LOD and LOQ reflected the acceptable sensitivity of the cited method.

Table 2. Validation results of the proposed UPLC method.

\begin{tabular}{cccc}
\hline Parameter & OMP & PPZ & LNZ \\
\hline $\begin{array}{c}\text { Accuracy (Mean * } \pm \text { SD) } \\
\text { Precision: }\end{array}$ & $99.65 \pm 1.21$ & $102.65 \pm 1.32$ & $101.32 \pm 0.95$ \\
Repeatability * & $101.23 \pm 0.72$ & $99.79 \pm 0.86$ & $101.44 \pm 1.15$ \\
Intermediate precision ${ }^{*}$ & $99.13 \pm 0.84$ & $100.34 \pm 0.61$ & $100.67 \pm 0.79$ \\
\hline Robustness: & & & \\
Mobile phase & $101.41 \pm 0.85$ & $99.26 \pm 0.89$ & $100.79 \pm 0.92$ \\
composition change & $99.76 \pm 0.88$ & $101.31 \pm 1.38$ & $101.22 \pm 0.95$ \\
pH change & $101.95 \pm 1.34$ & $101.46 \pm 1.18$ & $99.57 \pm 0.89$ \\
Flow rate change & & & \\
\hline Linearity: & $10-200$ & $10-200$ & $10-200$ \\
Range (ng/mL) & 97.116 & 52.406 & 94.542 \\
Slope & 1.4526 & 9.1226 & 7.1121 \\
Intercept & 0.9999 & 0.9998 & 0.9997 \\
\hline Correlation coefficient $(\mathrm{r})$ & 2 & 2 & 2 \\
\hline LOD (ng/mL) & 10 & 10 & 10 \\
\hline LOQ (ng/mL) & & &
\end{tabular}

\subsection{Method Application}

The cited method was effectively tested for quantification of the cited drugs by spiking them in distilled and tap water samples followed by the application of the optimized techniques of sample preparation and quantification. The results are given in Table 3.

Table 3. Determination of OMP, PPZ, and LNZ in spiked water samples using the proposed method.

\begin{tabular}{cccc}
\hline Specimen & OMP & PPZ & LNZ \\
\hline Distilled water (Rec. $\% \pm$ SD) ${ }^{*}$ & $102.52 \pm 0.79$ & $99.44 \pm 1.14$ & $99.98 \pm 0.92$ \\
Tap water (Rec. $\% \pm$ SD) ${ }^{*}$ & $101.04 \pm 0.88$ & $102.78 \pm 1.34$ & $101.69 \pm 0.97$ \\
\hline *Average of five measurements. & & &
\end{tabular}

Additionally, actual wastewater samples were carefully treated using the optimized sample preparation technique, then they were chromatographed by applying the optimized chromatographic parameters (Figure 2). The results were compared to those obtained by analyzing the actual wastewater samples using reference methods for the determination of OMP, PNT, and LNZ $[9,17,25]$ after sample pretreatment by the same sample preparation protocol (Table 4). Method performance for the determination of the cited drugs was also validated by the application of the standard addition procedure. The recovery $\%$ was calculated for the added pure PPI concentrations (Table 5). 


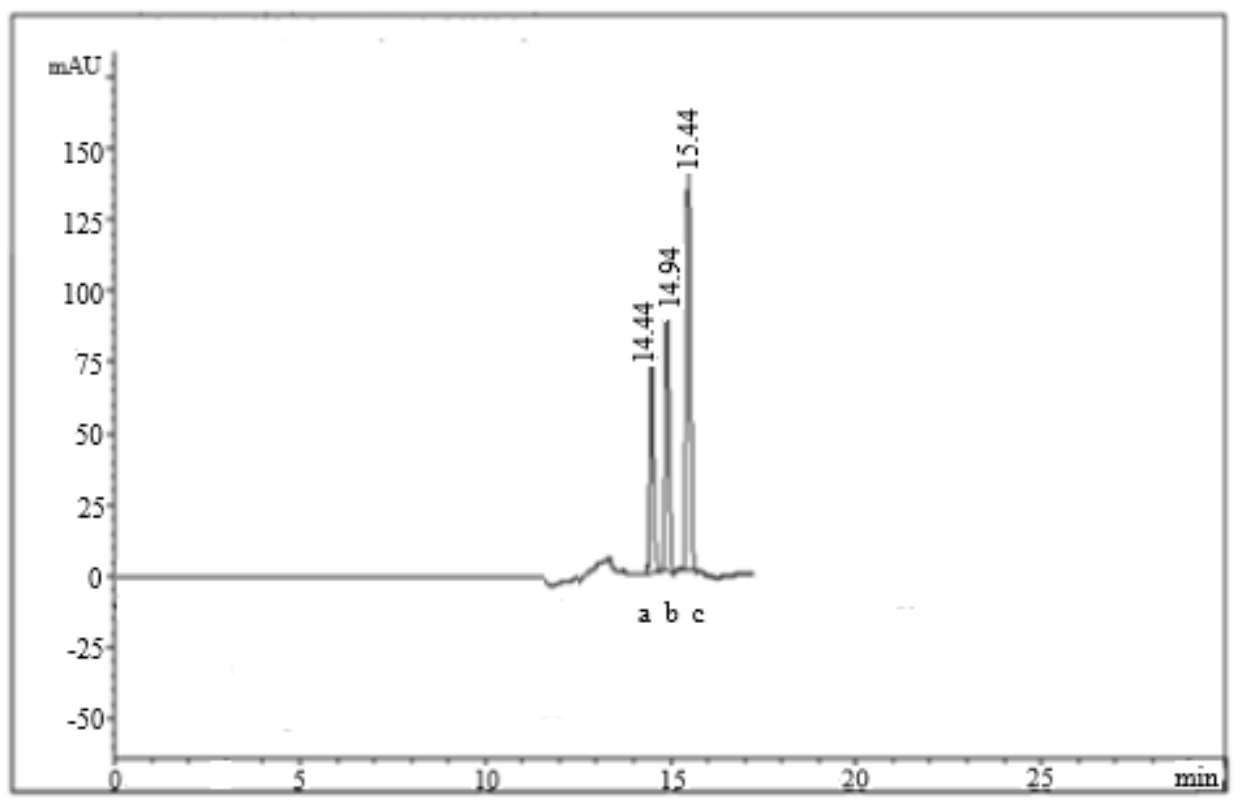

Figure 2. UPL chromatogram for the separation pattern of OMP (a), PNT (b), and LNZ (c) in actual wastewater sample.

Table 4. Determination of OMP, PPZ, and LNZ in actual wastewater samples from industrial pharmaceutical plants.

\begin{tabular}{ccccccc}
\hline \multirow{2}{*}{$\begin{array}{c}\text { Sample } \\
\text { Number }\end{array}$} & UPLC-Method & $\begin{array}{c}\text { Reference } \\
\text { Method [9] }\end{array}$ & UPLC-Method & $\begin{array}{c}\text { Reference } \\
\text { Method [17] }\end{array}$ & UPLC-Method & $\begin{array}{c}\text { Reference } \\
\text { Method [25] }\end{array}$ \\
\cline { 2 - 7 } & 20.02 & 19.98 & 30.55 & 30.59 & 15.72 & 15.43 \\
Sample 1 & 25.23 & 25.23 & 21.13 & 21.77 & 31.79 & 31.69 \\
Sample 2 & 31.67 & 31.89 & 20.56 & 20.91 & 40.01 & 39.99 \\
Sample 3 & 39.87 & 39.58 & 40.56 & 40.22 & 21.68 & 21.59 \\
Sample 4 & 22.97 & 22.46 & 25.37 & 25.12 & 41.83 & 41.11 \\
Sample 5 & & & & & PNZ $^{*}$ & \\
\hline
\end{tabular}

* Concentrations are calculated in nanograms per milliliter.

Table 5. Application of standard addition procedure for PPIs determination.

\begin{tabular}{cccc}
\hline \multirow{2}{*}{$\begin{array}{c}\text { Added PPIs } \\
(\mathbf{n g} / \mathbf{m L})\end{array}$} & OMP & PPZ & LNZ \\
\cline { 2 - 4 } & $\mathbf{( R e c o v e r y ~} \mathbf{\%} \pm \mathbf{S D})^{*}$ & $\mathbf{~ ( R e c o v e r y ~} \% \pm \mathbf{S D )}^{*}$ & $\mathbf{~ ( R e c o v e r y ~} \% \pm \mathbf{S D})^{*}$ \\
\hline 10 & $101.67 \pm 0.523$ & $99.67 \pm 0.652$ & $100.89 \pm 0.452$ \\
20 & $98.45 \pm 0.967$ & $102.67 \pm 1.328$ & $99.23 \pm 0.891$ \\
30 & $99.89 \pm 0.698$ & $101.67 \pm 0.945$ & $101.12 \pm 0.723$ \\
\hline
\end{tabular}

* Average of five readings.

\section{Discussion}

There is no doubt that monitoring environmental pollutants is a crucial task for conserving human health. Pharmaceutical drugs reaching domestic water or plants can constitute a great danger to human health as they can expose the human body for a long time, which may lead to serious effects on the human body. The present work demonstrated the capability of the quantification of PPI residues in wastewater coming from the pharmaceutical industry using a simple and sensitive UPLC method to monitor the environmental levels of the studied drugs to avoid their harmful effects on the human body.

Method optimization was done to get the best separation pattern and optimum peak resolution. This task was confirmed via the introduction of a full system suitability scheme (Table 1). The capacity factor values for the studied PPIs ensured that there was adequate time for the studied drugs to interact with the stationary phase properly. The values 
of resolution factors ensured baseline to baseline peak separation and excellent peak resolution. At the same time, the values of $\mathrm{N}$ and HETP declared high column efficiency. The tailing factors' values showed acceptable peak symmetry.

Method validation parameters were calculated and presented in a full validation sheet (Table 2). It confirmed the high accuracy and precision of the suggested method. Additionally, the validation sheet declared excellent method robustness that ensured the lack of considerable effects of slight changes in mobile phase composition, $\mathrm{pH}$, and flow rate on the cited method performance. Meanwhile, an acceptable method sensitivity was declared by the linearity range, LOD, and LOQ. They were very suitable for the determination of the cited PPIs in the concentration levels supposed to be present in the actual wastewater samples coming from pharmaceutical industrial factories around the KSA.

The proposed sample preparation technique and the quantification procedure were successfully applied for the determination of the studied PPIs in both spiked distilled and tap water samples giving acceptable results that confirmed the efficiency of the sample preparation procedure and the accuracy of the suggested method (Table 3). The suggested method was also applied for the quantification of the studied drugs in actual wastewater samples obtained from different pharmaceutical industrial factories around the KSA. To validate the results obtained by the cited method, the found concentrations in the actual wastewater samples were compared with those obtained by applying the reference methods for the determination of the studied PPIs in the same samples $[10,18,26]$, declaring good matching between the results (Table 4). The standard addition technique was well applied for the actual wastewater samples, confirming the excellent performance of the cited method (Table 5). The monitoring of the studied drugs by the developed method was found to be simple, economic, and suitable for environmental analysis.

\section{Materials and Methods}

\subsection{Instruments}

Ultra-performance liquid chromatography was done with a Waters ${ }^{\mathrm{TM}}$ Acquity system (Milford, CT, USA) with column dimensions $100 \times 2.1 \mathrm{~mm}, 1.7 \mu \mathrm{m}$. The detector was a UV-visible wavelength detector (Waters, 2489, Milford, CT, USA). SPE was performed using Agilent Bondesil ODS manually packed cartridges (Santa Clara, Canada).

\subsection{Chemicals and Reagents}

OMP, PNT, and LNZ bulk powder were kindly supplied by Riyadh Pharma (Riyadh, Kingdom of Saudi Arabia) and their percentage purities were labeled as $100.16 \pm 0.36$, $100.17 \pm 0.41$, and $99.45 \pm 0.78$, respectively. Methanol (HPLC-grade, purity $\geq 99.9 \%$ ), acetonitrile (HPLC-grade, purity $\geq 99.9 \%$ ), and distilled water (HPLC-grade) were purchased from Sigma-Aldrich (St. Louis, MO, USA). Potassium dihydrogen orthophosphate (HPLCgrade) was supplied by Fischer Chemicals ${ }^{\mathrm{TM}}$ (Zürich, Switzerland). Sodium hydroxide solution (HPLC-grade, LiChropur ${ }^{\mathrm{TM}}, 49.0-51.0 \%$ ) was purchased from Merck (Darmstadt, Germany).

\subsection{Standard Solutions}

Stock standard solutions of OMP, PNT, and LNZ $(100 \mu \mathrm{g} / \mathrm{mL})$ were prepared by accurately and separately weighing $10 \mathrm{mg}$ of each bulk powder into separate 100-mL volumetric flasks. The powder was dissolved in each flask, and the volume was brought to $100 \mathrm{~mL}$ using methanol. Working solutions of the studied drugs $(1 \mu \mathrm{g} / \mathrm{mL})$ were prepared from stock standard solutions $(100 \mu \mathrm{g} / \mathrm{mL})$ by diluting $1 \mathrm{~mL}$ of each stock standard solution $(100 \mu \mathrm{g} / \mathrm{mL})$ to $100 \mathrm{~mL}$ using methanol as a diluting solvent.

\subsection{Method Optimization}

Different stationary phases and mobile phases were tried to ensure good system suitability parameters. 


\subsection{Method Validation}

The validation process was conducted according to the ICH-Q2B guiding protocol.

\subsubsection{Linearity}

Different aliquots $(1-20 \mu \mathrm{g})$ of the studied drugs were accurately and separately taken to a group of volumetric flasks (100-mL capacity). The volume of each flask was then completed with methanol to obtain a concentration of $10-200 \mathrm{ng} / \mathrm{mL}$ of each studied drug. Samples were then chromatographed using a Waters ${ }^{\mathrm{TM}}$ column $(100 \times 2.1 \mathrm{~mm}$, $1.7 \mu \mathrm{m}$, Milford, CT, USA). The mobile phase was methanol:0.05 M potassium dihydrogen phosphate buffer (adjusted to $\mathrm{pH} 7.5$ using $\mathrm{NaOH})(50: 50, v / v)$. The composition of the mobile phase was changed by increasing the percentage of methanol by $5 \%$ and decreasing the percentage of the buffer by $5 \%$ every $4 \mathrm{~min}$. A flow rate of $0.8 \mathrm{~mL} / \mathrm{min}$ was maintained during the whole chromatographic analysis. The detection was done at $230 \mathrm{~nm}$. Peak areas were plotted against concentration to get standard curves then the regression equations were computed.

\subsubsection{Accuracy}

The accuracy can be defined as the percent of the recovered analyte from a definite quantity [31]. Results coming from nine samples of concentrations $(50,60$, and $70 \mathrm{ng} / \mathrm{mL}$ of each studied drug) were analyzed using the procedure under linearity.

\subsubsection{Precision}

It can be expressed as inter and intra-day precision, which is reported as $\%$ relative standard deviation for several experiments that are statistically significant so three concentrations of each studied drug $(50,60$, and $70 \mathrm{ng} / \mathrm{mL})$ were analyzed three times within the same day (intra-day) or on three successive days (inter-day) then the results were documented as \%RSD.

\subsubsection{Detection and Quantification Limits}

These can give us an idea about the method sensitivity, where LOD is the lowest detectable concentration and LOQ is the minimum quantified concentration. They were determined mathematically [31].

\subsubsection{Robustness}

The robustness can be assessed by evaluating the effect of minor changes on the proposed procedure. This was done by changing the developing system composition by adding $1 \%$ acetonitrile to the used mobile phase. Additionally, the rate of flow varied by $\pm 0.1 \mathrm{~mL} / \mathrm{min}$.

\subsubsection{System Suitability}

A full system suitability protocol was prepared including different parameters indicating migration rates of the separated drugs (capacity factor), selectivity and resolution (resolution factors), column efficiency ( $\mathrm{N}$ and HETP), and peak symmetry (tailing factor).

\subsection{Method Application}

\subsubsection{Wastewater Sample Collection and Storage}

Wastewater samples were collected from different pharmaceutical industry sites around the KSA. Filtration of the samples was done using membrane filters made of nylon. The samples were then stored in a cool and dark place.

\subsubsection{Preparation of Actual Wastewater Samples}

The actual wastewater samples were treated by SPE. First, the cartridges' packing material was treated with six $\mathrm{mL}$ methanol and three $\mathrm{mL}$ water for pre-conditioning purposes. The samples were then vortex mixed for approximately $10 \mathrm{~s}$ to ensure sample 
homogeneity. The type and volume of eluting solvent and flow rate were optimized. A sample volume of $4 \mathrm{~mL}$ was loaded, followed by washing using $1 \mathrm{~mL}$ water twice to remove the unbound substances in each cartridge and reduce any interfering band in chromatograms. Finally, the studied drugs were eluted from the cartridge with $2 \mathrm{~mL}$ methanol. Twenty $\mu \mathrm{L}$ of each studied drug were then chromatographed.

\subsubsection{Determination of OMP, PNT and LNZ in Spiked Water Samples}

The extraction procedure was evaluated by spiking distilled and tap water samples with different amounts of the studied drugs to get concentrations of 30,40 , and $50 \mathrm{ng} / \mathrm{mL}$ of the studied PPIs. The spiked samples were subjected to the extraction protocol then the extracted samples were analyzed using the optimized chromatographic technique.

\subsubsection{Determination of OMP, PNT and LNZ in Actual Wastewater Samples}

Five actual wastewater samples were subjected to the optimized SPE procedure, then they were chromatographed following the optimized chromatographic conditions. Calibration plots were used to get the unknown concentrations. The obtained results were compared to those obtained by applying reference methods for the determination of OMP, PNT, and LNZ $[10,18,26]$ after sample pretreatment by the same extraction protocol. Additionally, the suggested method was validated by the application of the standard addition procedure to the actual wastewater samples at three concentration levels $(10,20$, and $30 \mathrm{ng} / \mathrm{mL}$ ). The recovery \% was calculated for the added pure PPI concentrations.

\section{Conclusions}

This work represents the optimization, validation, and application of UPLC method for the simple, sensitive, and accurate determination of OMP, PNT, and LNZ drug residues in Saudi pharmaceutical industrial wastewater. The cited method offers distinct advantages over the already published work. It has greater sensitivity and better peak resolution if compared to the conventional HPLC methods. Regarding its comparison with the LCMS/MS methods, the suggested method is more economic and does not need a special instrument assembly. The suggested method has critical importance in tracing, detecting, and quantification of the studied PPIs drug residues in the pharmaceutical wastewaters, where their presence may lead to adverse consequences on human health and society.

Author Contributions: Conceptualization, S.A.A.-G. and H.H.A.; data curation, S.A.A.-G., A.B.A. and H.H.A.; formal analysis, S.A.A.-G. and A.B.A.; investigation, S.A.A.-G., H.H.A. and A.B.A.; methodology, S.A.A.-G. and H.H.A.; resources, S.A.A.-G., H.H.A. and A.B.A.; software, S.A.A.G.; supervision, S.A.A.-G.; validation, S.A.A.-G., H.H.A., and A.B.A.; visualization, S.A.A.-G. and H.H.A.; writing —original draft, S.A.A.-G. and A.B.A.; writing-review and editing, S.A.A.-G. and H.H.A. All authors have read and agreed to the published version of the manuscript.

Funding: This research received no external funding.

Institutional Review Board Statement: Not applicable.

Informed Consent Statement: Not applicable.

Data Availability Statement: The datasets used and/or analyzed during the current study are available from the corresponding author on reasonable request.

Acknowledgments: The current work was supported by Taif University Researchers Supporting Project number (TURSP-2020/29), Taif University, Taif, Saudi Arabia.

Conflicts of Interest: The authors declare that they have no competing interests regarding this manuscript.

Sample Availability: The datasets used and/or analyzed during the current study are available from the corresponding author on reasonable request. 


\section{References}

1. Hagiwara, T.; Mukaisho, K.-I.; Nakayama, T.; Hattori, T.; Sugihara, H. Proton Pump Inhibitors and Helicobacter Pylori-Associated Pathogenesis. Asian Pac. J. Cancer Prev. 2015, 16, 1315-1319. [CrossRef] [PubMed]

2. Reidar, F.; Tom, C.M.; Helge, L.W. Adverse Effects of Proton Pump Inhibitors-Evidence and Plausibility. Int. J. Mol. Sci. 2019, 20, 5203.

3. Pauline, D.; Shinya, I. Toxicity of Long-Term Use of Proton Pump Inhibitors in Children. Arch. Dis. Child. 2018, 103, 78-82.

4. Ali, M.M.; Ibrahim, E.A.; Abdalaziz, M.N. Development and Validation for RP-HPLC Method of Assay of Omeprazole Capsules Formulation. Am. J. Chem. Eng. 2018, 6, 1. [CrossRef]

5. Bhandage, A.; Bhosale, A.; Kasture, A.; Godse, V.P. Extractive Spectrophotometric Determination of Omeprazole in Pharmaceutical Preparations. Trop. J. Pharm. Res. 2009, 8, 449-454. [CrossRef]

6. Kalyankar, T.; Wadher, S.J.; Gholve, R.; Anitha, K. UV Spectrophotometric Estimation of Diclofenac Potassium and Omeprazole Magnesium in Bulk and Combined Tablet Dosage Form. Int. J. MediPharm Res. 2016, 2, 42-53.

7. Sanjana, C.J.; Shetty, A.S.; Ahmed, M.; Krishna, C.A.V.; Kumar, S.M.A. Development and Validation of Spectrophotometric Methods for Simultaneous Estimation of Omeprazole and Aspirin in Pharmaceutical Dosage Form. World J. Pharm. Pharm. Sci. 2018, 7, 1335-1348.

8. Hussein, R.F.; Binhashim, N.H.; Alvi, S.N.; Hammami, M.M. A Validated Reversed Phase HPLC Assay for the Determination of Omeprazole in Human Plasma. Eur. J. Pharm. Med. Res. 2016, 3, 26-30.

9. Sudha, T.; Reddy, K.K.; Hemalatha, P.V.; Ravikumar, V.R. An lc-ms/ms method for the determination of omeprazole on proton pump inhibitor in human plasma. Indones. J. Pharm. 2016, 27, 80. [CrossRef]

10. Rao, P.V.; Reddy, C.K.S.; Kumar, M.R.; Rao, D.D. Simultaneous determination of omeprazole and domperidone in capsules and in vitro dissolution studies by using stability indicating uplc. J. Liq. Chromatogr. Relat. Technol. 2012, 35, 2322-2332. [CrossRef]

11. Jadhav, S.B.; Kumar, C.K.; Bandichhor, R.; Bhosale, P. Development of RP UPLC-TOF/MS, stability indicating method for omeprazole and its related substances by applying two level factorial design; and identification and synthesis of non-pharmacopoeial impurities. J. Pharm. Biomed. Anal. 2016, 118, 370-379. [CrossRef]

12. Brinda, M.N.; Krishna Reddy, V.; Goud, E.S. Development and Validation of Ultra Performance Liquid Chromatographic Method for Assay of Omeprazole Blend. Int. J. Pharm. Bio. Sci. 2014, 4, 38-44.

13. Devi, O.Z.; Basavaiah, K.; Revanasiddappa, H.D.; Vinay, K.B. Titrimetric and spectrophotometric assay of pantoprazole in pharmaceuticals using cerium(IV) sulphate as oxidimetric agent. J. Anal. Chem. 2011, 66, 490-495. [CrossRef]

14. Salama, F.; El-Abasawy, N.; Razeq, S.A.; Ismail, M.; Fouad, M. Validation of the spectrophotometric determination of omeprazole and pantoprazole sodium via their metal chelates. J. Pharm. Biomed. Anal. 2003, 33, 411-421. [CrossRef]

15. Peres, O.; Oliveira, C.; Barrientos-Astigarraga, R.; Rezende, V.; Mendes, G.; De Nucci, G. Determination of Pantoprazole in Human Plasma by LC-MS-MS Using Lansoprazole as Internal Standard. Arzneimittelforschung 2004, 54, 314-319. [CrossRef] [PubMed]

16. Bhaskara, B.; Kumar, U.A.; Basavaiah, K. Sensitive liquid chromatography-tandem mass spectrometry method for the determination of pantoprazole sodium in human urine. Arab. J. Chem. 2011, 4, 163-168. [CrossRef]

17. Challa, B.R.; Boddu, S.H.; Awen, B.Z.; Chandu, B.R.; Bannoth, C.K.; Khagga, M.; Kanala, K.; Shaik, R.P.; Challa, B.S.R. Development and validation of a Sensitive bioanalytical method for the quantitative estimation of Pantoprazole in human plasma samples by LC-MS/MS: Application to bioequivalence study. J. Chromatogr. B 2010, 878, 1499-1505. [CrossRef] [PubMed]

18. Devi, O.Z.; Basavaiah, K.; Vinay, K.B. Quantitative determination of lansoprozole in capsules and spiked human urine by spectrophotometry through ion-pair complex formation reaction. J. Saudi Chem. Soc. 2013, 17, 387-396. [CrossRef]

19. Rahman, N.; Bano, Z.; Azmi, H.; Kashif, M. A kinetic spectrophotometric method for the determination of lansoprazole in pharmaceutical formulations. J. Serbian Chem. Soc. 2006, 71, 1107-1120. [CrossRef]

20. Rizwana, I.; Prakash, K.V.; Mohan, G.K. Extractive Spectroscopic Determination of Lansoprazole in Pharmaceutical Dosage Form. Chem. Sci. Trans. 2014, 3, 1390-1395.

21. Kumar, S.M.; Kumar, D.S.; Rajkumar, T.; Kumar, E.U.; Suba, G.A.; Diwedi, D. Development and Validation of RP-HPLC Method for the Estimation of Lansoprazole in Tablet Dosage Form. J. Chem. Pharm. Res. 2010, 2, 291-295.

22. Yanfei, L.; Lishuang, X.; Ming, X.; Jia, F.; Xing, T. A Validated, Specific, Stability-Indicating HPLC Method for Determination of Lansoprazole Enteric Capsules and Related Impurities. Asian J. Pharm. Sci. 2012, 7, 149-154.

23. Singh, S.; Choudhary, N.; Rai, J.; Sharma, S.I.; Yadav, A.; Gautam, H.; Chaturvedi, S.; Agrawal, V. Validated RP-UPLC Method Development for Estimation of Lansoprazole in Tablet Dosage Form. Int. J. Pharm. Sci. Drug Res. 2013, 5, $105-107$.

24. Singh, S.; Choudhary, N.; Rai, J.; Inamullah, S.; Sharma, S. A Validated RP-UPLC Method Development for Simultaneous Estimation of Lansoprazole and Naproxen in Bulk and Tablet Dosage Form. Asian J. Pharm. Clin. Res. 2013, 6, $150-152$.

25. Ganeshab, M.; BhagiyaLakshmia, M.; Hemalathaa, P.; Rahul, P.; Sakthimanigandand, K.; Hyun, T.J.; Rajasekarb, K. Liquid chromatography-electrospray tandem mass spectrometry (lc-ms/ms) determination of lansoprazole in human plasma. J. Liq. Chromatogr. Relat. Technol. 2011, 2, 129-142. [CrossRef]

26. López-Roldán, R.; de Alda, M.L.; Gros, M.; Petrovic, M.; Martín-Alonso, J.; Barceló, D. Advanced monitoring of pharmaceuticals and estrogens in the Llobregat River basin (Spain) by liquid chromatography-triple quadrupole-tandem mass spectrometry in combination with ultra performance liquid chromatography-time of flight-mass spectrometry. Chemosphere 2010, 80, 1337-1344. [CrossRef] [PubMed] 
27. Pedrouzo, M.; Borrull, F.; Pocurull, E.; Marcé-Recasens, R.M. Presence of Pharmaceuticals and Hormones in Waters from Sewage Treatment Plants. Water Air Soil Pollut. 2010, 217, 267-281. [CrossRef]

28. Gómez, M.J.; Malato, O.; Ferrer, I.; Agüera, A.; Fernández-Alba, A.R. Solid-phase extraction followed by liquid chromatographytime-of-flight-mass spectrometry to evaluate pharmaceuticals in effluents. A pilot monitoring study. J. Environ. Monit. 2007, 9, 718-729. [CrossRef]

29. Bueno, M.J.M.; Hernando, M.D.; Herrera, S.; Gómez, M.J.; Fernández-Alba, A.R.; Bustamante, I.; García-Calvo, E. Pilot survey of chemical contaminants from industrial and human activities in river waters of Spain. J. Environ. Anal. Chem. 2010, 90, 321-343. [CrossRef]

30. Lucie, N.; Ludmila, M.; Petr, S. Advantages of application of UPLC in pharmaceutical analysis. Talanta 2006, 68, 908-918.

31. International Conference on Harmonisation of Technical Requirements for Registration of Pharmaceuticals for Human Use. In ICH Harmonized Tripartite Guideline, Validation of Analytical Procedures: Text and Methodology Q2 (B); PharmaLogica, Inc.: Charlotte, NC, USA, 2005. 